\title{
Pokies and poverty: problem gambling risk factor geography in New Zealand
}

\author{
Benedict W. Wheeler ${ }^{\mathrm{a}}$, Janette E. Rigby ${ }^{\mathrm{b}, *}$, Terry Huriwai ${ }^{\mathrm{c}}$ \\ ${ }^{a}$ Public Health Intelligence Applications Laboratory, School of Earth Sciences, Victoria University of Wellington, P.O. Box 600, \\ Wellington, New Zealand \\ ${ }^{\mathrm{b}}$ Department of Geography, University of Sheffield, Winter Street, Sheffield S10 2TN, UK \\ ${ }^{\mathrm{c}}$ Māori Mental Health, Mental Health Directorate, Ministry of Health, P.O. Box 3877, Christchurch, New Zealand
}

Accepted 29 October 2004

\begin{abstract}
Up to $2 \%$ of adults in New Zealand can be considered problem gamblers, where the activity has an impact on the well-being of those who gamble, and often their close associates. The most common activity involves non-casino gaming machines (NCGMs). This paper explores the geography of gambling 'opportunity' at small-area scales, and finds excess provision in those areas classified as highly deprived. Geographically weighted regression has been used to investigate the possibility that the degree of inequity in NCGM provision varies across New Zealand. As machines are licensed, this provides an opportunity for policy implementation towards risk reduction.
\end{abstract}

(C) 2004 Elsevier Ltd. All rights reserved.

Keywords: Gambling; Addiction; Deprivation; Ethnicity; Policy

\section{Introduction}

The impacts on health and well-being of 'addictive' behaviours such as tobacco smoking, alcohol consumption and drug use are increasingly well-established and researched, though health policy often lags behind research findings, and appears increasingly entrenched in individual responsibility and 'victim blaming' interpretations. Less widely researched is the issue of problem gambling, most likely to be expressed in the context of mental health, and often linked with other dependencies (Abbott and Volberg, 1999).

Gambling is a popular, common leisure activity which can range from a personal, small, wager between two individuals to very high stakes at the blackjack or

\footnotetext{
*Corresponding author. Tel.: + 441142227911 ; fax: +441142797912 .
}

roulette table in a casino: indeed, casino gambling is portrayed by the media as a glamorous social activity. The reasons why people gamble beyond a casual leisure activity are complex and contested. Whilst problem gambling is generally viewed as a continuum (Abbott, 2002), in its most extreme form it has been viewed as an addiction, and hence medicalised: in 1997 pathological gambling was included in the International Classification of Diseases (ICD9) coding, and hence recognised as an official psychiatric disorder. A substantial body of the current research into problem gambling follows the medical model, based within the discipline of psychology.

At present the impacts of problem gambling on health and well-being follow two not-unrelated pathways. The first is within mental health, where studies demonstrate relatively high rates of depression, schizophrenia, lifethreatening behaviour and suicide among problem 
gamblers. In an epidemiological study based in St. Louis, USA, Cunningham-Williams and colleagues (1998) found significantly elevated odds ratios for major depression and schizophrenia in problem gamblers, alongside suicidal tendencies. DeCaria et al. (1996) also observed high rates of a wide range of mental health problems in problem gamblers. In 2002, $10 \%$ of users of an established telephone helpline for problem gambling in New Zealand reported suicide ideation, with 30 of the 4655 clients having attempted suicide in the past year (Paton-Simpson et al., 2003). More women than men reported loneliness and isolation in connection with the development of a gambling habit, (Grant and Kim, 2002); the majority of respondents reported significant family histories of gambling problems and alcohol dependence. The second pathway relating to co-dependence on alcohol means that it is often difficult to explore problem gambling as a separate issue.

'Problem gambling refers to patterns of gambling behaviour that compromise, disrupt or damage health, personal, family or vocational pursuits.' (Abbott and Volberg, 2000, p. 11). The individual gambler is most likely to feel the most severe effects, but these can impact on close family members, friends and workplace colleagues. The gambling process can often take priority over other commitments and everyday routines, and where the gambling is sustained over many hours, the gambler will neglect eating and sleeping, resulting in poor physical health (Christensen et al., 2001). Problem gamblers report poor self-related health, and high rates of depression, anxiety and stress (Pasternak and Fleming, 1999; Sullivan et al., 1998). Studying pathological gamblers, Grant and Kim (2002) noted that $15.4 \%$ of the women and $13.2 \%$ of the men reported stress or anxiety as a trigger for gambling. The mean annual percentage incomes lost as a result of this gambling were $83.2 \%$ for women and $54.3 \%$ for men, though it should be noted that these respondents were at the extreme of the gambling spectrum.

Although problem gambling can develop with all of the possible gambling opportunities available, the most commonly identified source is non-casino gaming machines (NCGMs), commonly known as slot machines one-armed bandits or, in New Zealand particularly, 'pokies'. Around $80 \%$ of clients using problem gambling support services report problems primarily related to use of gaming machines (Paton-Simpson et al., 2003).

New Zealand has been at the forefront of research on the prevalence of gambling and problem gambling: a national study in 1991 was the first to use an internationally validated instrument to assess problem gambling (Abbott and Volberg, 1991). A subsequent study in 1999 (Abbott and Volberg, 2000) found that approximately $94 \%$ of adults in had participated in gambling at some time. This is similar to figures from Australia, Canada and Sweden, but is higher than the
USA. It was estimated that $10.5 \%$ are regular gamblers, a decrease from $18 \%$ in 1991, and half the proportion in Australia. Between $0.6 \%$ and $1.1 \%$ of New Zealand adults could be classified as current problem gamblers, and a further $0.3-0.7 \%$ could be classified as current probable pathological gamblers (the $0.7 \%$ represents some 20,100 in urgent need of support services). The profile of those who were over-represented experiencing problems relating to NCGMs were those who were male, employed but with no degree or higher educational qualifications, under 35, Māori, and never married.

Recognition of the adverse social impacts of problem gambling is reflected in the fact that the Ministry of Health has now taken responsibility for problem gambling service provision within the remit of its Mental Health Directorate, and that the 2002 Health Survey of New Zealand has, for the first time, included questions on gambling activities. A different government department, the Department of Internal Affairs, retains responsibility for the licensing of pokies devolved to the regional level of Territorial Local Authorities, and hence there is no national policy relating to the provision of 'opportunity'.

This raises two questions: the (equitable) extent of provision of 'opportunity' i.e. where are pokies located, and are there many predictable social characteristics of the neighbourhood. Further, where are support services located with respect to perceived need? At present, the confidential process offered by the support services means that no detailed data are consistently collected on the residential address of the person seeking help, and therefore this latter question is not addressed here.

If NCGMs are indeed most easily accessible in communities most at risk of developing gambling problems, this suggests that easy accessibility to gambling opportunities may increase the risk of problem development, and hence may help to explain the overrepresentation of Māori and Pacific People in problem gambling service usage statistics. Additionally, it may indicate that NCGM operators place machines in communities that they know will make the greatest use of them, despite the resultant increased risk of problematic gambling. If this is the case, then there could be potential to reduce the public health impact of problem gambling through planning controls and other regulations that actively seek to limit the siting of machines in vulnerable communities.

\section{Methods}

This investigation assesses ecological associations between small area population characteristics and the location of NCGMs. Data regarding the prevalence or incidence of problem gambling are not available for 
small areas of New Zealand. For this reason, the analyses presented here rely upon the prevalence of known risk factors and how they relate to the location of gaming machines. As outlined above, socio-economic deprivation and Māori or Pacific ethnicities have been identified specifically as risk factors for problem gambling in New Zealand. From a geographical perspective it is therefore of interest to investigate the hypothesis that gaming machines may be more easily accessible in deprived areas or those with larger populations of the ethnic groups identified. New Zealand's Asian population is not over-represented in problem gambling service utilisation figures. However, it is suggested that there is a 'disproportionate level of participation in gambling by people of Asian appearance or from Asian countries' (Wong and Tse, 2003). The Asian population is therefore also considered in analyses that follow.

Data were obtained on the location of NCGMs, and on the deprivation and ethnicity characteristics of the population. These data were allocated to census area units (CAUs), census output areas commonly used for small area analysis. There were approximately 1800 CAUs at the 2001 census, with a mean population of around 2000. Deprivation data consisted of the 2001 NZDep deprivation index (Salmond and Crampton, 2002), constructed from census-based measures including income, unemployment, amenity access and education, but not ethnicity. The NZDep index is frequently divided into deciles, each containing approximately one tenth of the total number of area units. Decile 1 indicates the least deprived $10 \%$ of all CAUs, whilst decile 10 indicates the most deprived $10 \%$ of all CAUs.

Ethnicity data were available from 2001 census statistics supplied by Statistics New Zealand. Prioritised counts $^{1}$ of people self-identifying with Māori, Pacific and Asian ethnicities were obtained for each CAU. Since problem gambling is most prevalent in the young adults age group (Paton-Simpson et al., 2003), and older age groups are dominated by NZ Europeans given longer

\footnotetext{
${ }^{1}$ Prioritisation is a systematic means used by Statistics NZ to deal with the fact that individuals filling in a census form may identify with more than one ethnic group. Summing the total response for each ethnicity results in multiple counting and a total greater than the total population. Prioritisation is used to allocate a single ethnic group to each individual, and these counts by ethnicity do sum to $100 \%$. These prioritised counts can therefore be used to calculate ethnicity population proportions for each CAU without multiple counting of individuals. A small degree of error results, since allocating someone to a single ethnicity, despite their identification with more than one group, will result in undercounting the other groups. However, the prioritisation system is designed to prioritise Māori and Pacific groups, and since these are the groups of interest here, the error is deemed acceptable.
}

life expectancy (Ajwani et al., 2003), ethnicity proportions were calculated for the age range 15-39 only.

A database of non-casino gaming machine venues held by the Department of Internal Affairs (DIA, the regulatory and licensing body) was obtained as at 31 March 2003. This database details the address of each venue (mostly pubs and clubs) along with the number of machines licensed, to the maximum of 18 . The data are self-reported by the societies that run the machines, and are not comprehensively verified by DIA. However, these data do provide the only spatially referenced listing of NCGMs across New Zealand. The venues database was address-geocoded to produce a mappable dataset (there are no postcodes or zip code equivalents in New Zealand). Spatial analyses and mapping were carried out using ArcGIS (ESRI, 2003).

Each venue was allocated to the CAU in which it fell; a standard 'point-in-polygon' GIS operation. This allowed the total number of venues and gaming machines in each CAU to be calculated. However, this approach can result in boundary effects due to the arbitrary, political nature of CAU boundaries and also to any inaccuracies in the address geocoding process. Small adjustments to a boundary or corrections to a point location could see a venue being allocated to a neighbouring area. Ideally, we would know the 'sphere of influence' of each venue, and relate this area to underlying census areas. However, no information or estimates regarding venue catchment areas are available, and investment in travel time analysis was not deemed appropriate given a) the lack of evidence for acceptable travel-to-venue times and b) that this is not comparable to a journey-to-service analysis; we are primarily interested in the area characteristics of the immediate neighbourhood of the venue. A simple buffering approach was therefore used to complement the pointin-polygon analysis. The buffering process involves constructing a circle around each venue (point) and overlaying these circles on the CAU polygons. Using this process we can select CAUs that fall within the specified distance of a venue and compare them to those outside of this distance. The selection of buffer radius here is arbitrary, and the circle produced is an estimate of the 'neighbourhood' of a venue. Various buffers from 250 to $5 \mathrm{~km}$ were used in order that sensitivity analyses could be carried out.

A potential source of error in area-based geographical analyses such as this is the Modifiable Areal Unit Problem or MAUP (Openshaw, 1983). This problem occurs when associations observed using a set of spatial units do not hold when alternative spatial aggregations are applied to the same data. To investigate whether or not the MAUP may be an issue here, analyses were also carried out using Census meshblocks, the most detailed spatial unit for New Zealand census data output (in 2001, there were approximately 38,000 meshblocks with 
mean population around 100). The smaller populations associated with meshblocks can make analyses more unstable, but they are useful in providing an insight into whether or not any conclusions drawn from CAU analyses are applicable using an alternate spatial framework.

Once the data had been assembled into the relevant areal units, simple cross-tabulations and graphs were constructed. Regression models were then used to estimate associations between NCGM accessibility, deprivation and ethnicity. Māori and Pacific Peoples are over-represented in the most deprived areas (see the next section). The regression models allowed us to measure reciprocally adjusted associations between ethnicity/NCGM location and deprivation/NCGM location. The count of machines allocated to a CAU was used as the dependent variable in a Poisson regression model, with an offset for the CAU population.

Given the variation of deprivation and ethnicity characteristics across the country, and the potential for the various local authorities to influence NCGM siting differently, it is possible that associations between these variables may vary spatially. To investigate this 'nonstationarity', geographically weighted regression $^{2}$

\footnotetext{
${ }^{2}$ GWR is used to investigate spatial non-stationarity in relationships between geographic variables. Instead of assuming that the association between variable $x$ and variable $y$ is fixed and can be represented by a single set of (global) regression parameters, the association is allowed to vary across space. An illustrative example often quoted is the relationship between house characteristics and house prices. For instance, we might expect that in areas far from public open spaces, larger gardens may be associated with substantial increases in house price. In areas close to a public park, the association between garden size and house price may be smaller (i.e. the regression slope may be less steep). In the context of this investigation, we are interested in whether global regression parameters are a good representation of the association between deprivation, ethnicity and gambling opportunity, or if these associations vary across New Zealand and are better modelled using spatially varying regression coefficients.

The process of model calibration for GWR involves carrying out a local regression model centred on each point in the dataset, using the surrounding datapoints as the observation subset for each local model. Resultant regression parameters, such as beta-coefficients, uncertainty measures and $R^{2}$ values, are then attached to the central point of that local model (the calibration point) and can be mapped. Each observation in a local model is weighted with respect to the distance between itself and the calibration point. Points closest to the calibration point are assigned the greatest weight, whilst those further away carry less weight. Hence, the regression model is 'geographically weighted'. The model 'kernel' refers to the weighting function used to select and weight the subset of observations for each calibration point, and the 'bandwidth' indicates the extent of the subset of datapoints to be used for each local model. Using GWR 3.0, the kernel bandwidth may be fixed (Gaussian weight function, selects all datapoints within
}

Table 1

CAU descriptive statistics - prioritised ethnicity (see Footnote 1) proportions for population aged $15-39(n=1695)$

\begin{tabular}{lcc}
\hline Prioritised ethnicity & Mean & Median \\
\hline \% Māori & 17.0 & 11.8 \\
\% Pacific peoples & 4.1 & 1.5 \\
\% Asian & 5.3 & 2.5 \\
\% NZ European/Pakeha & 68.6 & 74.7 \\
\hline
\end{tabular}

(GWR) was used to assess spatial variation in regression coefficients. The software GWR 3.0 (Fotheringham et al., 2003) was used to calibrate models comparable to the global Poisson model described above, in order to investigate spatial variations in the observed relationships.

\section{Findings}

\section{Descriptive statistics}

All results are presented for analyses of mainland CAUs (North and South Islands only) with non-zero population, since these constraints are imposed for GWR analyses. Table 1 presents descriptive statistics for the ethnicity proportion variables in order to give some idea of the relative proportions of these broad ethnic groups (with the caveat that using prioritised ethnicity results in a varying degree of under-estimation). NZ Europeans (Pakeha) form the largest ethnic group, followed by Māori, Asian and Pacific populations. However, these proportions do vary widely across the country; substantial Māori populations reside in northern and north-eastern parts of the North Island, whilst Pacific and Asian populations are highly urbanised throughout.

Table 2 indicates the proportion of CAUs whose boundaries fall within the specified distance of an

\section{(footnote continued)}

a set distance of the calibration point) or adaptive (Bi-square weight function, selects all points up to the specified number of nearest neighbours). Adaptive kernels are often used where the spacing between datapoints is irregular and a fixed distance would result in significant variation in local model sample sizes.

Once the GWR model has been run, tests can be used to compare it to the global model in order to indicate whether or not observed associations exhibit non-stationarity. Along with the approximation tests described in Findings, GWR 3.0 is able to implement Monte Carlo simulation tests to test for an overall improvement in model fit compared to the global model. However, this test is currently only available for ordinary least squares regression and not for the logistic or Poisson models used here. 
NCGM venue, based on the various buffer distances. Around half of the area units actually contain an NCGM venue, and almost all CAUs (96\%) are within $5 \mathrm{~km}$ of at least one venue. The larger buffers encompass high proportions of CAUs, making comparison of areas within and outside the buffers meaningless. This endorses the suggestion above that this analysis should simply consider the immediate neighbourhood of each venue.

To illustrate associations between deprivation and ethnicity, Fig. 1 shows the distribution of NZDep deciles for CAUs in the top quintile of each ethnicity proportion variable. This clearly illustrates that areas

Table 2

Number and proportion of census area units within specified distance of a non-casino gaming machine venue

\begin{tabular}{lrl}
\hline CAUs with an NCGM venue & $n$ & $\%$ Of CAUs \\
\hline Within boundary & 882 & 51.5 \\
Within $250 \mathrm{~m}$ & 1170 & 68.4 \\
Within $500 \mathrm{~m}$ & 1317 & 77.0 \\
Within $1 \mathrm{~km}$ & 1475 & 86.2 \\
Within $2 \mathrm{~km}$ & 1581 & 92.4 \\
Within $5 \mathrm{~km}$ & 1650 & 96.4 \\
\hline
\end{tabular}

with the highest proportions of Māori or Pacific populations have highly deprived populations (if these CAUs had the same deprivation characteristics as the whole country, each decile would contain roughly the same number of CAUs). In total, around $56 \%$ of Māori and $72 \%$ of Pacific Peoples live in CAUs in deciles 8, 9, and 10; the most deprived 30\% of CAUs.

It is clear from Fig. 2 that there is a much greater provision of NCGMs in the more deprived CAUs in New Zealand. These charts illustrate the disproportionate location of machines in the most deprived areas, with $53 \%$ of machines in the most deprived $30 \%$ of CAUs (deciles 8, 9 and 10). This is particularly true of those CAUs in deciles 8 and 9; perhaps the financial circumstances, which characterise decile 10 would prohibit much 'leisure' activity which incurs a cost. Fig. 2 (b) illustrates that the distribution is not simply due to the population distribution amongst the deciles; the pattern of NCGMs per head of population across the deciles is very similar to that of the total count of NCGMs. However, we do need to be cautious with interpretation here, as the NZDep scores characterise the areas, and not those individuals or households who live within them.

Hence, we see that NCGMs are much more likely to be found in the most deprived areas of New Zealand,
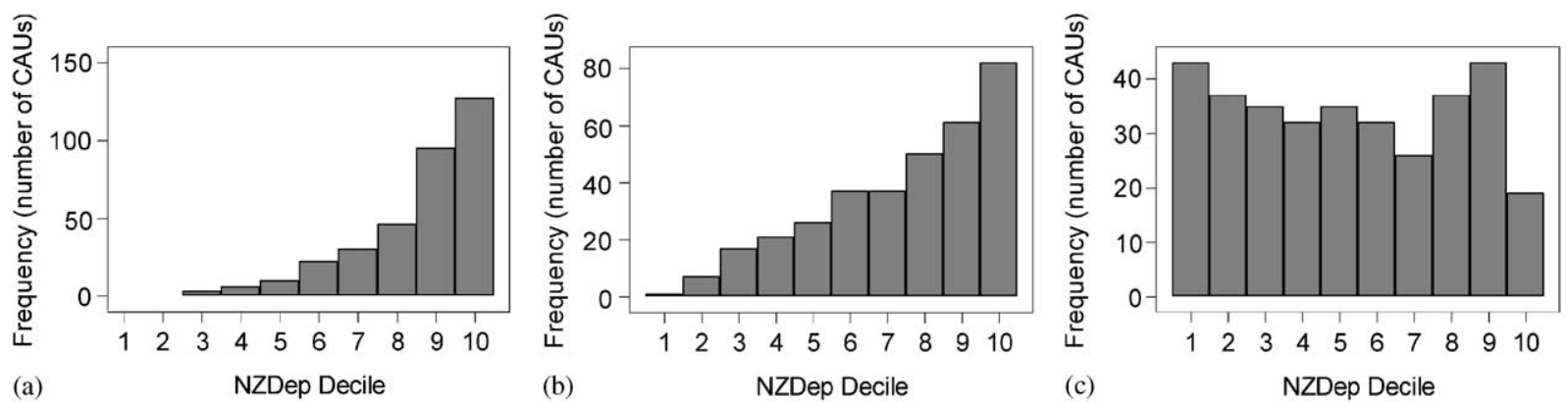

Fig. 1. Distribution of deprivation index deciles for CAUs in the top quintile of each ethnic group proportion variable (a) \% Māori (b) $\%$ Pacific (c) \% Asian.
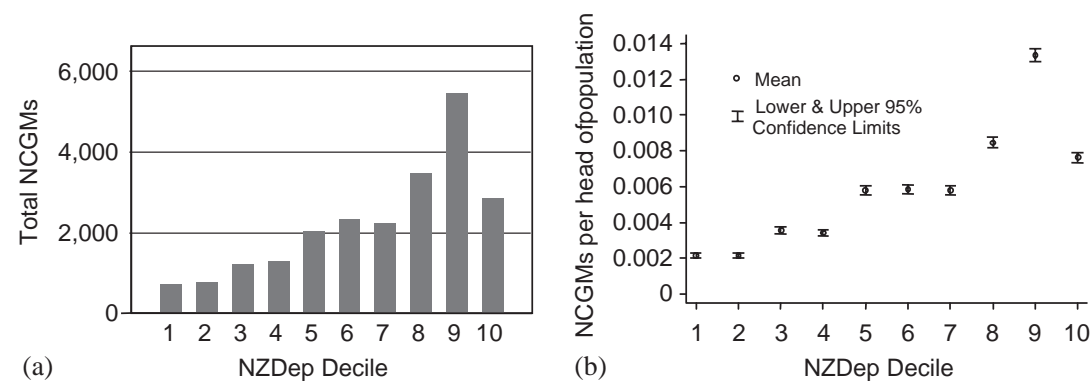

Fig. 2. Distribution of non-casino gaming machines across NZDep deprivation index deciles $(1=$ least deprived; $10=$ most deprived): (a) Total machines by NZDep decile. (b) Poisson proportion (machines per head of population) and $95 \%$ confidence intervals. 
and that Māori and Pacific populations are more likely to reside in deprived areas. The regression analyses that follow investigate these associations simultaneously.

\section{Global regression analyses}

Results from the Poisson model predicting machine numbers with NZDep decile and ethnicity percentages are presented in Table 3. The results indicate that rate ratios across NZDep deciles do not increase in a linear fashion, and a likelihood ratio test indicated that this categorical, rather than linear, approach produced a better model fit. The rate ratios indicate that, for example, a CAU in NZDep decile 8 is likely to have about 7 times the density of gaming machines (per head of population) compared to a CAU in decile 1, after adjustment for the ethnicity variables included. Higher proportions of Asian population are also associated

Table 3

Poisson regression results $(n=1695$ CAUs $)$

\begin{tabular}{cccr}
\hline Explanatory variable & $\begin{array}{c}\text { Rate } \\
\text { ratio }\end{array}$ & & $p 5 \%$ CI \\
\hline $\begin{array}{c}\text { (Least deprived) } \\
\text { NZDep decile 1 }\end{array}$ & 1.00 & - & - \\
2 & 1.03 & $(0.93,1.14)$ & 0.552 \\
3 & 1.79 & $(1.63,1.96)$ & $<0.001$ \\
4 & 1.85 & $(1.69,2.03)$ & $<0.001$ \\
5 & 3.29 & $(3.02,3.59)$ & $<0.001$ \\
6 & 3.72 & $(3.42,4.05)$ & $<0.001$ \\
7 & 4.13 & $(3.79,4.50)$ & $<0.001$ \\
8 & 6.90 & $(6.35,7.50)$ & $<0.001$ \\
9 & 12.83 & $(11.81,13.93)$ & $<0.001$ \\
\% & 15.13 & $(13.77,16.62)$ & $<0.001$ \\
\% Māori aged 15-39 & 0.98 & $(0.98,0.98)$ & $<0.001$ \\
\% Pacific aged 15-39 & 0.95 & $(0.95,0.95)$ & $<0.001$ \\
\% Asian aged 15-39 & 1.01 & $(1.01,1.01)$ & $<0.001$ \\
\hline
\end{tabular}

Rate ratios refer to the relative density of non-casino gaming machines per head of population, comparing each NZDep decile to decile 1 , and for an increase of $1 \%$ in the ethnicity variables. with higher machine densities, but higher proportions of Māori and Pacific populations are associated with lower machine densities.

For reasons explained below, comparable Poisson models could not be run using geographically weighted regression. Instead, logistic models were run, and results from the equivalent global logistic model are presented here for comparison. The dependent variable in this case is not machine density, but a binary variable indicating presence/absence of a venue within (or within distance of) a CAU. This reduces the data, in that the number of machines is no longer considered. However, this method also allows for a simple buffer distance sensitivity analysis, repeating the model with binary independent variables based on buffers of differing sizes.

Table 4 presents illustrative results showing the effect of changing buffer size on regression coefficients. Since $77 \%$ of CAUs are within $500 \mathrm{~m}$ of a venue, rising to $96 \%$ within $5 \mathrm{~km}$ (see Table 2), analyses using dependent variables based on the larger buffers are not useful. However, results for the point-in-polygon, $250 \mathrm{~m}$ buffer and $500 \mathrm{~m}$ buffer values are consistent. The only major change with buffer size is that the odds ratio (OR) for the Pacific population variable becomes greater than unity for buffers $1 \mathrm{~km}$ and over. At this distance, $86 \%$ of CAUs are selected as being in proximity to a venue. Given that the Pacific population is highly urbanised, this finding is unsurprising, since most bars and clubs will be found in population centres and these are mostly covered by the larger buffers. The $250 \mathrm{~m}$ buffer was selected as the most appropriate indicator of a CAU being in immediate proximity to a venue, and full results of the model using this response variable are presented in Table 5.

The results of the logistic regression model are comparable to those for the Poisson regression presented above. Higher deprivation deciles are associated with greater probability of being in proximity (within $250 \mathrm{~m}$ ) of an NCGM venue, as is having a higher Asian population percentage. Higher percentages of Māori and Pacific populations are associated with lower odds of being in proximity to a venue, although in this case

\section{Table 4}

Illustrative results from global logistic regression models indicating the effect of varying buffer size around NCGM venues ( $p$-value for all ORs $<0.001$ unless otherwise stated)

\begin{tabular}{llllll}
\hline Explanatory variable & \multicolumn{2}{l}{ Pokie venue proximity: ORs } \\
\cline { 2 - 6 } & $\begin{array}{l}\text { Venue within } \\
\text { CAU }\end{array}$ & $\begin{array}{l}\text { Venue within } \\
\text { 250 m of CAU }\end{array}$ & $500 \mathrm{~m}$ & $1 \mathrm{~km}$ & $2 \mathrm{~km}$ \\
\hline NZDep decile 10 versus decile 1 & 13.48 & 8.25 & 11.96 & 6.14 & $2.73(p=0.13)$ \\
\% Māori & 0.97 & 0.97 & 0.96 & 0.97 & 0.97 \\
\% Pacific & 0.97 & $0.98(p=0.04)$ & $0.99(p=0.55)$ & $1.04(p=0.15)$ & $1.26(p=0.03)$ \\
\% Asian & $1.01(p=0.22)$ & 1.05 & 1.06 & 1.19 & 1.71 \\
\hline
\end{tabular}


the Pacific population coefficient is of borderline statistical significance.

In order to investigate the possibility of the modifiable areal unit problem, comparable logistic and Poisson models to those above were constructed for equivalent data at meshblock level. Regression coefficients (not presented here for the sake of brevity) were very similar in sign and magnitude to those produced by the CAU models, giving reassurance that these findings are unlikely to be an artefact of the areal units selected.

\section{Geographically weighted regression}

Geographically weighted Poisson regression models could not be run using these data. This is because a large number of CAUs are allocated a zero count of machines

Table 5

Logistic regression results ( $n=1695$ CAUs). ORs refer to the relative odds of the CAU being within $250 \mathrm{~m}$ of a pokie venue

\begin{tabular}{llllr}
\hline Explanatory variable & OR & $95 \%$ CI & \multicolumn{1}{c}{$p$} \\
\hline (Least deprived) NZDep decile 1 & 1.00 & & - \\
& 2 & 1.58 & $(1.02,2.43)$ & 0.039 \\
& 3 & 1.99 & $(1.28,3.10)$ & 0.002 \\
& 4 & 2.50 & $(1.60,3.91)$ & $<0.001$ \\
& 5 & 3.72 & $(2.32,5.96)$ & $<0.001$ \\
& 6 & 5.46 & $(3.32,9.00)$ & $<0.001$ \\
& 7 & 4.46 & $(2.73,7.28)$ & $<0.001$ \\
& 8 & 5.60 & $(3.33,9.40)$ & $<0.001$ \\
(Most deprived) decile 10 & 9 & 7.22 & $(4.05,12.89)$ & $<0.001$ \\
\% Māori aged 15-39 & 6.18 & $(3.05,12.49)$ & $<0.001$ \\
\% Pacific aged 15-39 & & 0.98 & $(0.97,0.99)$ & $<0.001$ \\
\% Asian aged 15-39 & 0.99 & $(0.97,1.00)$ & 0.078 \\
& 1.04 & $(1.03,1.06)$ & $<0.001$ \\
\hline
\end{tabular}

by the point-in-polygon method. This results in some local data subsets having zero Poisson events (where none of the CAUs in a local model contain a venue), making these data unsuitable for the process that optimises local model sample size. However, using the binary dependent variable based on the $250 \mathrm{~m}$ buffer as above, logistic models equivalent to those described above could be run using GWR.

Each CAU's centroid $x, y$ co-ordinates were used as the locational references in GWR models. Models were run separately for the North and South Islands, given the physical barrier existing between them (the Cook Strait). Processes available within GWR 3.0 were used to optimise the local model bandwidth using an adaptive kernel, in order to account for the highly variable distance between CAU centroids (see Footnote 2).

Running logistic models in GWR 3.0, two approximation tests have been recommended to assess whether or not the GWR model is a better fit than the global regression model (Fotheringham, 2003, personal communication). GWR calculates the Akaike Information Criterion (corrected) (AICc, a measure of goodness of fit) for the global model and for the local GWR models. If the AICc decreases, the model fit is improved. Secondly, regression coefficients from the global and local models are compared. If the quantity $(2 *$ standard error) for the global model parameter is less than the inter-quartile range for the equivalent local model parameter, the local model is probably a better fit. Additionally, the optimised bandwidth gives an indication of the degree of spatial non-stationarity - a lower bandwidth suggests greater spatial variation.

Table 6 presents summary figures for North and South Island GWR models comparable to the global logistic model in Table 5. Since the AICc is lower for the North Island global model than the respective GWR

Table 6

Summary information from logistic GWR models for North and South Island CAUs (independent variable = presence/absence of an NCGM within $250 \mathrm{~m}$ of CAU boundary)

\begin{tabular}{|c|c|c|c|c|}
\hline Model summary & Parameter & $\begin{array}{l}\text { Global model: } \\
2 * \text { coefficient } \\
\text { standard error }\end{array}$ & $\begin{array}{l}\text { Local models: } \\
\text { coefficient inter- } \\
\text { quartile range }\end{array}$ & $\begin{array}{l}\text { IQR }>2 * \text { standard } \\
\text { error }\end{array}$ \\
\hline North Island & Intercept & 0.308 & 0.129 & No \\
\hline Optimised bandwidth: 1146 & \% Māori 15-39 & 0.012 & 0.007 & No \\
\hline (of 1186 CAUs) & $\%$ Pacific $15-39$ & 0.014 & 0.004 & No \\
\hline Global AICc: 1432.8 & $\%$ Asian 15-39 & 0.022 & 0.006 & No \\
\hline Local AICc: 1434.5 & NZDep Decile & 0.072 & 0.031 & No \\
\hline South Island & Intercept & 0.490 & 0.133 & No \\
\hline Optimised bandwidth: 408 & \% Māori 15-39 & 0.052 & 0.024 & No \\
\hline (Of 509 CAUs) & $\%$ Pacific $15-39$ & 0.158 & 0.617 & Yes \\
\hline Global AICc: 558.1 & \% Asian 15-39 & 0.062 & 0.021 & No \\
\hline Local AICc: 551.7 & NZDep Decile & 0.106 & 0.146 & Yes \\
\hline
\end{tabular}

$\mathrm{IQR}>2 * \mathrm{SE}$ indicates spatial non-stationarity in regression parameter. 
model, and the IQR is not greater than twice the standard error for any of the parameters, we can conclude that there is unlikely to be any spatial nonstationarity and the global model is a good fit for the North Island. However, for the South Island, the AICc decreases for the GWR model, and the IQR versus standard error measures suggest non-stationarity in the Pacific population and NZDep parameters. The GWR output file was used to map these parameters.

The maps in Fig. 3 show results of the South Island GWR model for the NZDep parameter. The OR associated with this parameter varies between 1.07 and 1.27 , and suggests that the greatest inequity in terms of NCGM siting and deprivation may be in the southern part of the island, around Dunedin, Invercargill and Queenstown. The lowest ORs are around Christchurch (though these are still indicative of inequity). It is difficult to calculate and demonstrate exact measures of statistical significance here. However, the map of $t$ values suggests, unsurprisingly, that the most reliable estimates are those for which the ORs are highest.

The maps in Fig. 4 show equivalent results for the \% Pacific population aged 15-39 parameter. Here, the odds ratio varies between 0.71 and 1.57 , suggesting that the direction of the association may vary across the island. The greatest inequity is found around the Christchurch area, and again $t$-values are also greatest in this area. Importantly, this result suggests that the global OR estimate of around 0.99 is inappropriate and not indicative of the association between Pacific population and gaming machine location everywhere. Again, it is difficult to infer statistical significance here, especially

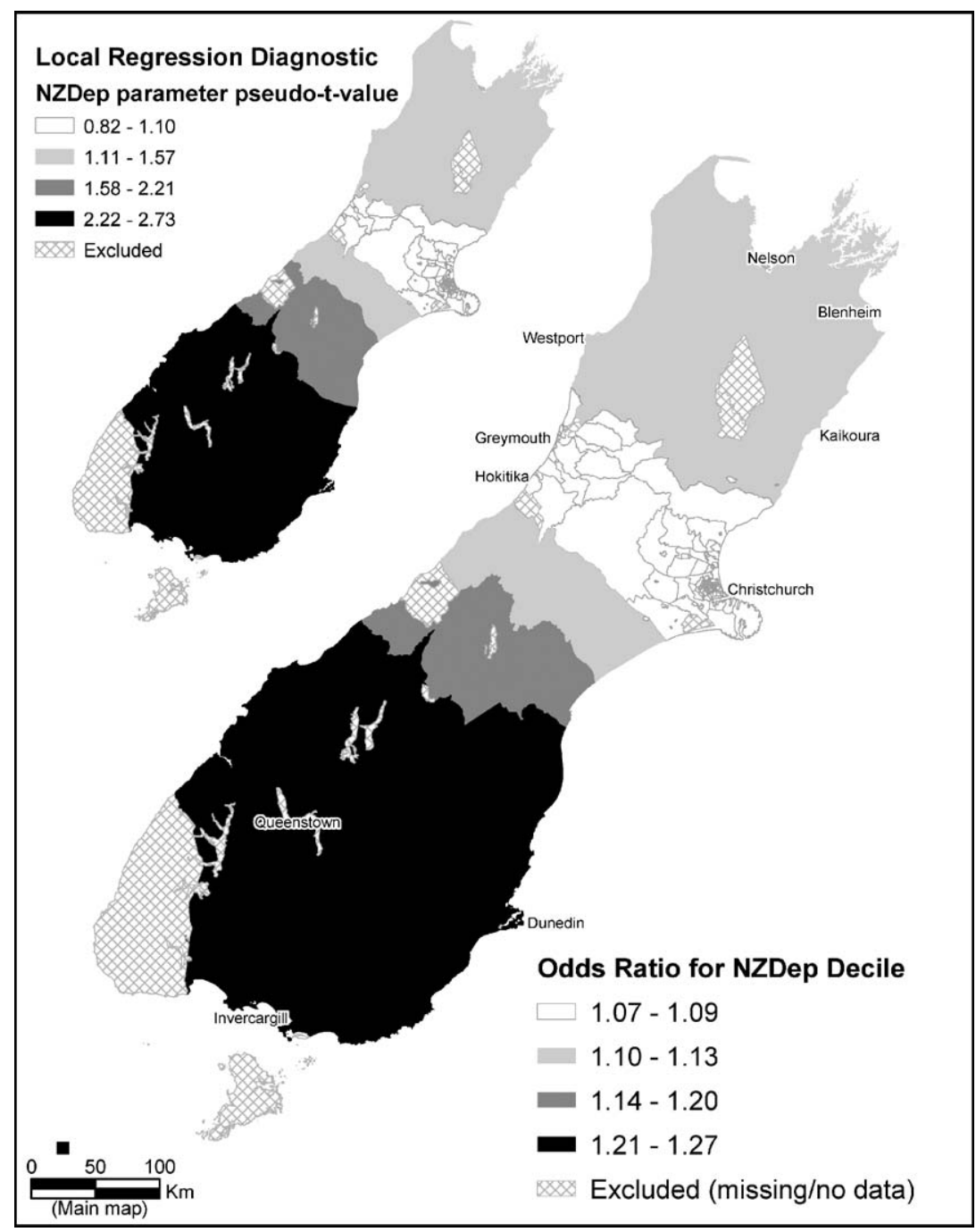

Fig. 3. Local model output for NZDep parameter from South Island logistic GWR model. 


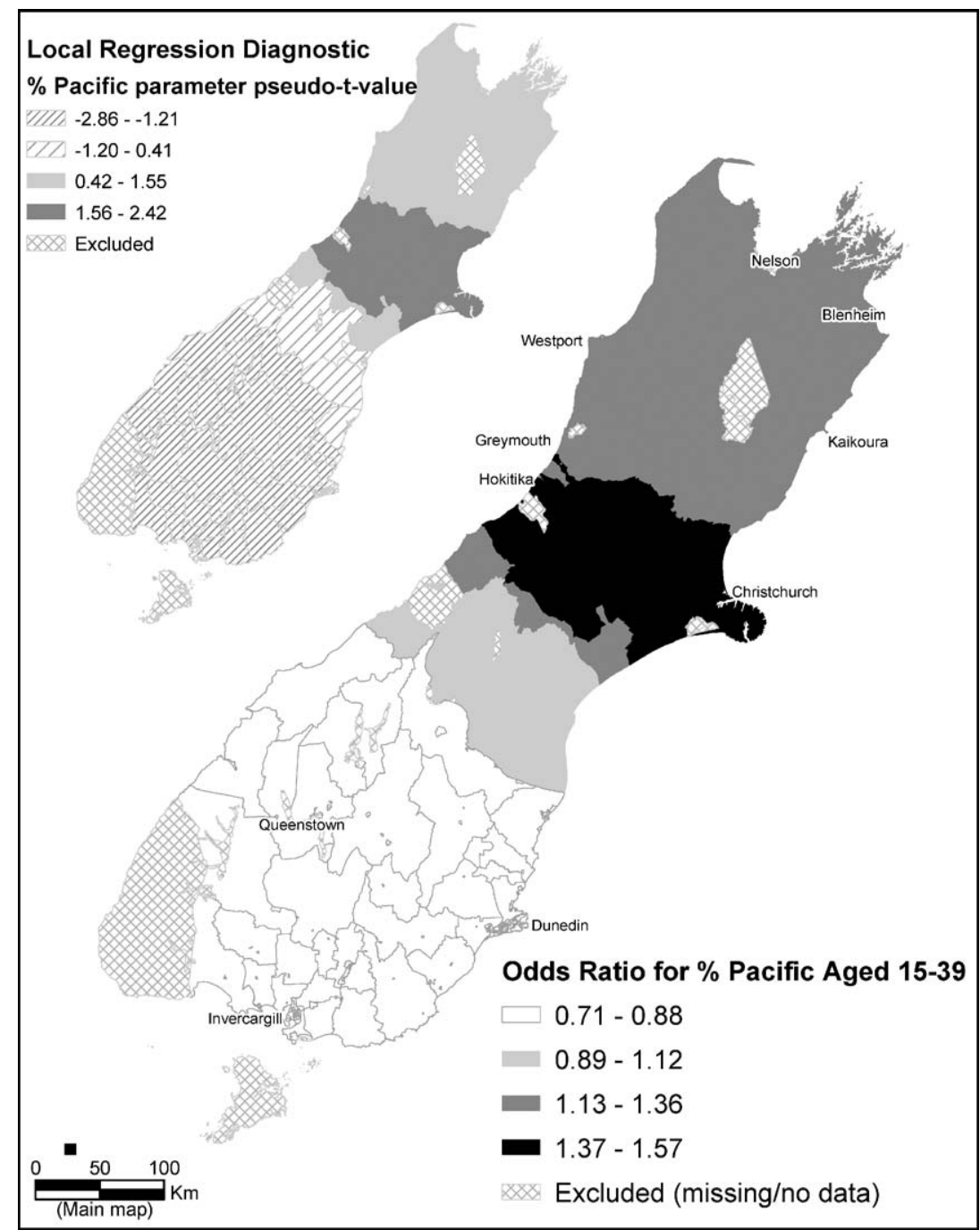

Fig. 4. Local model output for \% Pacific aged 15-39 parameter from South Island logistic GWR model.

that given in the global model the $p$-value for the $\%$ Pacific coefficient is only of borderline significance $(p=$ $0.078)$.

GWR models were also constructed for the meshblock-level data set. Given the increased number of spatial units being analysed, models took much longer to run (the North Island model took about 14 days on a $2.4 \mathrm{GHz}$ PC with a gigabyte of RAM). Given the increased spatial resolution, it is easier for tests to indicate variation in regression coefficients. Approximate test results suggested that all parameters exhibited spatial non-stationarity, although value ranges were very low, and not considered to be meaningfully different from the global result (for example the odds ratio inter-quartile range for the \% Māori parameter was $0.98-0.99$, and for the $\%$ Pacific parameter
0.97-0.98). We have therefore focused on results for CAUs here, in order to be consistent throughout.

\section{Conclusions}

There is growing concern for the impacts of problem gambling on individuals, their families and others in New Zealand. Recent legislation has mandated the NZ Ministry of Health to deal with problem gambling as a significant public health issue. In this paper, we have addressed the geographical dimensions of problem gambling risk according to the location of non-casino gaming machines, the primary cause of problems in the majority of individuals using counselling and treatment services. 
It is clear that gaming machines are disproportionately sited in the most deprived areas of New Zealand, potentially influencing or at least facilitating the development of problem gambling habits in those who can least afford them, and thus impacting adversely on wellbeing. The associations between machine location and ethnic communities are less clear, and in terms of the Māori and Pacific populations, not in the expected direction given the over-representation of these ethnic groups in service use statistics.

Geographically weighted regression models suggest that these associations are, in general, uniform across the nation. However, the South Island model does suggest that (a) inequity in terms of deprivation and machine siting may be greatest in the Southern area of the island and (b) the Pacific communities in the Christchurch area may be 'overexposed' to gaming machines. These findings warrant further investigation, and may help to shape the responsible gaming policies of the relevant local authorities.

Gaming machine operators, local planning authorities and central government have a role to play in determining whether or not the current and future placement of machines is appropriate, given knowledge of population risk factors. However, gaming organisations contribute substantial sums of money to charitable and community causes, and the positive benefits of these contributions are not disputed. The organisations also pay a levy that goes to directly fund problem gambling service provision. There are clearly ethical and moral dimensions to decisions about where and when gambling should be permitted. Further, gambling taxation is generally viewed as acceptable, and there is no great pressure on licensing authorities to reduce provision.

Limitations of this study include the inability to demonstrate cause and effect between gaming machine location and development of problem gambling. However, to do so would require detailed individual, qualitative investigation that is not within the remit of this study. Further work at the micro-level to further investigate the geographic elements of problem gambling on a more personal basis would be of merit. Additionally, the availability of problem gambling prevalence estimates (such as usage rates of the national problem gambling telephone helpline) for small areas would enable an ecological study such as this to investigate the geography of risk more thoroughly. In terms of limitations of the data used here, since the NCGM database was produced, new legislation has resulted in the removal of some machines from some venues with a newly imposed maximum of nine machines. This means that the machine counts for March 2003 are an overestimate of the present case. However, DIA estimate that the new legislation would only result in the immediate removal of around $6 \%$ of NCGMs, which is unlikely to have any substantial effect on analyses here. Future analyses may be able to look at whether or not reductions in machine numbers have been appropriately disproportionate (with the greatest reduction in deprived areas and subsequent reduction in inequity). It is possible that new limits on machine numbers per venue may simply result in a greater number of licensed venues within the same areas, defeating the object of the legislation. The GWR methodology helps to highlight the potential error in assuming that global statistics adequately describe circumstances across the entire nation. GWR is an exploratory technique, and as such is of limited inferential power. However, it does appear here that the technique is able to shed further light on interesting and sometimes contradictory findings.

\section{Acknowledgements}

We thank the anonymous reviewers for their helpful comments on an earlier version of this paper. Thanks to Ron King for providing a prior version of the gaming machine database, and to Kylie Mason for assisting with the geocoding of the version used here. Thanks to the Department of Internal Affairs for supplying the NCGM data; DIA is not responsible for any alterations to or analysis of the data here. Financial support for the study was provided by the Ministry of Health.

\section{References}

Abbott, M., 2002. Psychology, health and gambling. In: Curtis, B. (Ed.), Gambling in New Zealand. Dunmore Press, Palmerston North, pp. 135-141.

Abbott, M.W., Volberg, R.A., 1991. Gambling and problem gambling in New Zealand. Department of Internal Affairs, Wellington.

Abbott, M.W., Volberg, R.A., 1999. Gambling and Problem Gambling in the Community: An International Overview and Critique. Department of Internal Affairs, Wellington.

Abbott, M.W., Volberg, R.A., 2000. Taking the Pulse on Gambling and Problem Gambling in New Zealand: A report on Phase One of the 1999 National Prevalance Survey. Department of Internal Affairs, Wellington.

Ajwani, S., Blakely, T., Robson, B., Tobias, M., Bonne, M., 2003. Decades of Disparity: Ethnic mortality trends in New Zealand 1980-1999. Ministry of Health \& University of Otago, Wellington.

Christensen, M.H., Patsdaughter, C.A., Babington, L.M., 2001. Health care providers' experiences with problem gamblers. Journal of Gambling Studies 17, 71-79.

Cunningham-Williams, R.M., Cotter, L.B., Compton, W.N., Spitznagel, E.L., 1998. Taking chances: problem gamblers and mental health disorders - results from the St Louis Epidemiologic Catchment Area Study. American Journal of Public Health 88, 1093-1096.

DeCaria, C.M., Hollander, E., Grossman, R., Wong, C.M., Mosovich, S.A., Cherkasky, S., 1996. Diagnosis, neurobiology 
and treatment of pathological gamblers. Journal of Clinical Psychiatry 57 (Suppl. 8), 80-83.

ESRI, 2003. ArcGIS 8.3. Environmental Systems Research Institute, Redlands.

Fotheringham, A.S., Brunsdon, C., Charlton, M., 2003. GWR 3.0. University of Newcastle, Newcastle-upon-Tyne.

Grant, J.E., Kim, S.W., 2002. Gender differences in pathological gamblers seeking medication treatment. Comprehensive Psychiatry 43, 56-62.

Openshaw, S., 1983. The Modifiable Areal Unit Problem. Geo Books, Norwich.

Pasternak, A.V., Fleming, M.F., 1999. Prevalence of gambling disorders in a primary care setting. Archives of Family Medicine 8, 515-520.
Paton-Simpson, G.R., Gruys, M.A., Hannifin, J.B., 2003. Problem gambling counselling in New Zealand: 2002 National Statistics. Problem Gambling Purchasing Agency, Palmerston North.

Salmond, C., Crampton, P., 2002. NZDep2001 Index of Deprivation. Wellington School of Medicine and Health Sciences, Wellington.

Sullivan, S., Arroll, B., Coster, G., Abbott, M., 1998. Problem gamblers: a challenge for GPs. New Zealand Family Physician 25, 37-42.

Wong, J., Tse, S., 2003. The face of Chinese migrants' gambling: A perspective from New Zealand. http:// www.camh.net/egambling/issue9v1/opinion/wong/index. html Accessed: 15/1/2004. 